\title{
Structural Phase Transitions in $\left[\left(\mathrm{C}_{2} \mathrm{H}_{5}\right)_{4} \mathrm{~N}\right]_{3} \mathrm{Sb}_{2} \mathrm{Br}_{9}$ and $\left[\left(\mathrm{C}_{2} \mathrm{H}_{5}\right)_{4} \mathrm{~N}_{3} \mathrm{Bi}_{2} \mathrm{Br}_{9}\right.$
}

\author{
J. Zaleski, R. Jakubas, Z. Galewski, and L. Sobczyk \\ Institute of Chemistry, University of Wrocław, Poland \\ Z. Naturforsch. 44a, $1102-1106$ (1989); received May 11, 1989
}

\begin{abstract}
Dielectric, DSC and preliminary X-ray diffraction studies on $\left[\left(\mathrm{C}_{2} \mathrm{H}_{5}\right)_{4} \mathrm{~N}\right]_{3} \mathrm{Sb}_{2} \mathrm{Br}_{9}$ (TEABA) and $\left[\left(\mathrm{C}_{2} \mathrm{H}_{5}\right)_{4} \mathrm{~N}\right]_{3} \mathrm{Bi}_{2} \mathrm{Br}_{9}$ (TEABB) are reported. The compounds are isomorphous, space group $\mathrm{P} 6_{3} 22$. The static electric permittivity measurements on single crystals in the temperature range $220-290 \mathrm{~K}$ revealed that both compounds show two distinct dielectric anomalies; TEABA at $T_{c 1}=258 \mathrm{~K}$ and $T_{c 2}=252 \mathrm{~K}$, while TEABB at $T_{c 1}=265 \mathrm{~K}$ and $T_{c 2}=248 \mathrm{~K}$. The DSC studies show only one anomaly of first order at $T_{\mathrm{c} 1}=258 \mathrm{~K}$ for TEABA, and $T_{\mathrm{c} 1}=266 \mathrm{~K}$ for TEABB. These transitions are presumably related to a freezing of the reorientational motion of the tetraethylammonium cations.
\end{abstract}

Key words: Alkylammonium halogenoantimonates, Bismuthates, Phase transition, Dielectric, DSC, Single crystals.

\section{Introduction}

Considerable attention has recently been given to various halogenoantimonates and bismuthates of the general formula $\left[\left(\mathrm{CH}_{3}\right)_{n} \mathrm{NH}_{4-n}\right]_{3} \mathrm{M}_{2} \mathrm{X}_{9}(n=1,2,3,4$; $\mathrm{M}=\mathrm{Sb}, \mathrm{Bi} ; \mathrm{X}=\mathrm{Cl}, \mathrm{Br})$. These compounds exhibit interesting properties; some of them show transitions to polar phases, cf. [1] and references cited therein. So far only compounds with small cations have been studied. It seemed interesting to study also salts with large cations, such as tetraethylammonium (TEA) ions.

Crystallographic data are available for several TEA salts. In many cases the TEA cations are disordered at room temperature $[2,3]$, thus one can expect structural phase transitions (SPT) on lowering the temperature. So far SPTs in (TEA) ${ }_{2} \mathrm{MX}_{4}(\mathrm{M}=\mathrm{Co}, \mathrm{Cu}, \mathrm{Ni}$; $\mathrm{X}=\mathrm{Cl}, \mathrm{Br}$ ) [4], (TEA) ${ }_{2} \mathrm{SnCl}_{6}$ [5] and (TEA)FeX ${ }_{4}$ $(\mathrm{X}=\mathrm{Cl}, \mathrm{Br})[6]$ were found by calorimetry. SPTs in TEA salts were also studied by NMR [7], IR and Raman [8] methods, however there are only two $[9,10]$ reports on the dielectric behaviour of those salts.

We have investigated the dielectric properties of single crystals of two isomorphous compounds (TEA) ${ }_{3} \mathrm{Sb}_{2} \mathrm{Br}_{9}$ (tetraethylammonium nonabromodiantimonate (TEABA)) and (TEA) ${ }_{3} \mathrm{Bi}_{2} \mathrm{Br}_{9}$ (tetraethylammonium nonabromodibismuthate (TEABB)).

So far there exists only some information about the Raman and FIR spectra of polycrystalline TEABA $[11,12]$ but non for TEABB.

Reprint requests to $\mathbf{J}$. Zaleski, Institute of Chemistry, University of Wrocław, Joliot Curie 14, 50-383 Wrocław, Poland.

\section{Experimental}

The TEABA (TEABB) salts were prepared by adding $\mathrm{Sb}_{2} \mathrm{O}_{3}\left((\mathrm{BiO})_{2} \mathrm{CO}_{3}\right)$ to a concentrated solution of $\mathrm{HBr}$. After $\mathrm{Sb}_{2} \mathrm{O}_{3}\left((\mathrm{BiO})_{2} \mathrm{CO}_{3}\right)$ had dissolved, a stoichiometric amount of an aqueous solution of tetraethylamine bromide was added. The precipitation occurred immediately. The precipitates were dissolved in an excess of hot $\mathrm{HBr}$. Upon cooling the yellow crystals were filtered off and dried. The single crystals were grown by a slow evaporation of the nitromethane solution at constant room temperature. The composition of the compounds was confirmed by elemental analysis for $\mathrm{C}, \mathrm{H}$, and $\mathrm{N}$.

The salts crystallize as hexagonal transparent plates. The single crystals should be kept in a saturated solution and only withdrawn before an experiment because they tend to decompose. This process starts from the surfaces and is much faster for TEABB.

The dielectric permittivities and losses were measured at $1 \mathrm{kHz}(E=5 \mathrm{~V} / \mathrm{cm})$ by a Meratronic automatic capacitance bridge meter type E $315 \mathrm{~A}$. The measurements were performed with a cooling/heating rate of $0.1 \mathrm{~K} / \mathrm{min}$. The dimensions of the plates were approximately $5 \times 5 \times 1 \mathrm{~mm}^{3}$.

The DSC measurements were performed on a Rikagu calorimeter (scanning speed $5 \mathrm{~K} / \mathrm{min}$ ).

The preliminary X-ray diffraction studies were carried out using a Weissenberg goniometer with $\mathrm{CuK}_{\alpha}$ radiation at room temperature. 


\section{Results and Discussion}

The Weissenberg photographs show that TEABA and TEABB are isomorphous and crystallize hexagonally with the Laue symmetry $6 / \mathrm{mm}$. The only systematic absences were $000 l, l=2 n$, which are characteristic of $\mathrm{P} 6_{3} 22$ space group. The structural parameters are $a=10.3, \quad c=24.4 \AA, \quad V=2242 \AA^{3}, \quad Z=2, \quad d_{c}=2.02$, $d_{m}=2.00 \mathrm{~g} / \mathrm{cm}^{3}$ for TEABA and $a=10.3, c=24.6 \AA$, $V=2260 \AA^{3}, \quad Z=2, \quad d_{c}=2.25, \quad d_{m}=2.23 \mathrm{~g} / \mathrm{cm}^{3}$ for TEABB.

There exists no information about the crystallographic structure of the compounds but the vibrational Raman and FIR spectra for TEABA suggest that the anionic sublattice of the crystal is composed of discrete $\mathrm{Sb}_{2} \mathrm{Br}_{9}^{3-}$ units [11]. Such anions would be thus built of two face sharing $\mathrm{SbBr}_{6}$ bioctahedra with three bridging and six terminal bromine atoms.

In Figs. 1 and 2 the temperature dependencies of the dielectric permittivity of TEABA are shown along the $c$ and $a$-axes, respectively. On cooling from room temperature $\varepsilon_{c}^{\prime}$ decreases nearly linearly (Fig. 1) down to $T_{c 1}=258 \mathrm{~K}$, where it drops significantly (from 15.5 to 13.2) in a temperature range of $4 \mathrm{~K}$. A small thermal hysteresis around $T_{c 1}$ is observed $(1-1.5 \mathrm{~K})$. The character of $\varepsilon_{a}^{\prime}(T)$ (Fig. 2) is significantly different from that of $\varepsilon_{c}^{\prime}(T)$. On cooling $\varepsilon_{c}^{\prime}(T)$ exhibits a broad maximum centered around $T_{c 2}=252 \mathrm{~K}\left(\varepsilon_{a(\max )}^{\prime}=18.2\right)$ and afterwards decreases to 15.1 at $220 \mathrm{~K}$. On heating a significant thermal hysteresis of about $2.5-3 \mathrm{~K}$ near $T_{c 2}$ occurs.

The $\varepsilon_{c}^{\prime}(T)$ and $\varepsilon_{a}^{\prime}(T)$ plots show that in the studied temperature range there are two distinct anomalies of $\varepsilon^{\prime}(T)$. The shape of the anomaly round $T_{c 1}$ (Fig. 1) is characteristic of a first order transition while the order of the transition at $T_{c 2}$ is not clear. The shape of the anomaly in Fig. 2 is characteristic of second order, whereas the thermal hysteresis rather suggests first order character.

The unusual anisotropy of dielectric behaviour should be pointed out. The transition at $T_{c 2}$ is not discernible in the $\varepsilon_{c}^{\prime}(T)$ plot although it is well pronounced as a high peak in the plot of $\varepsilon^{\prime}$ along the $a$ direction. Vice versa the transition at $T_{c 1}$ is not visible in the $\varepsilon_{a}^{\prime}(T)$ plot but well reflected as a jump in the $\varepsilon_{c}^{\prime}(T)$ plot.

An anomaly of the dielectric loss $(\tan \delta)$ was not observed in the studied temperature range, neither in the $c$ nor in the $a$ direction.

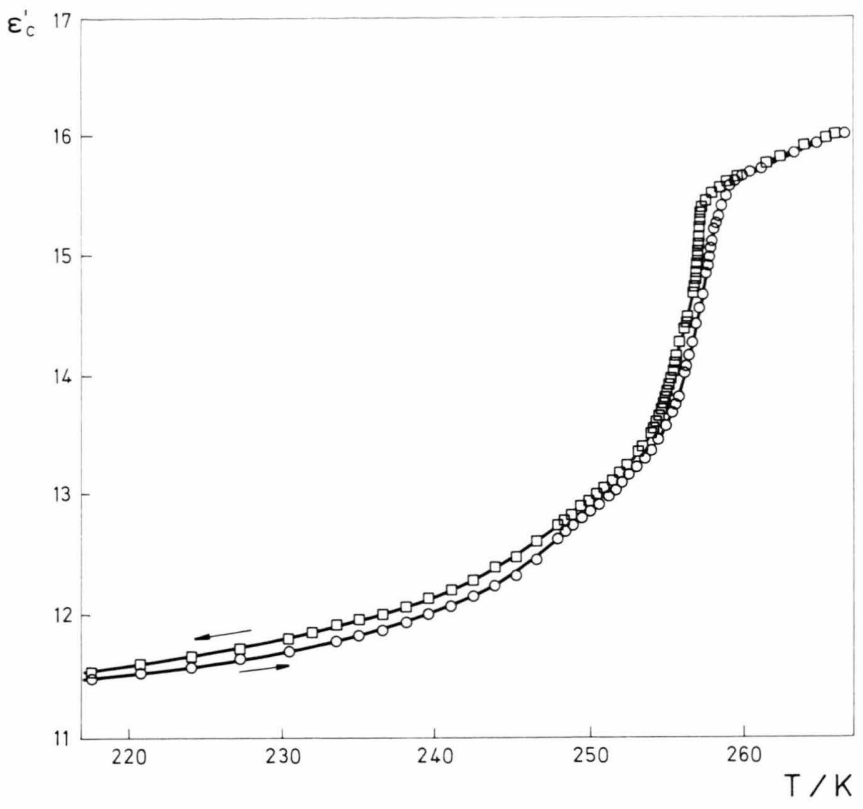

Fig. 1. Electric permittivity versus temperature for TEABA along $c$-axis.

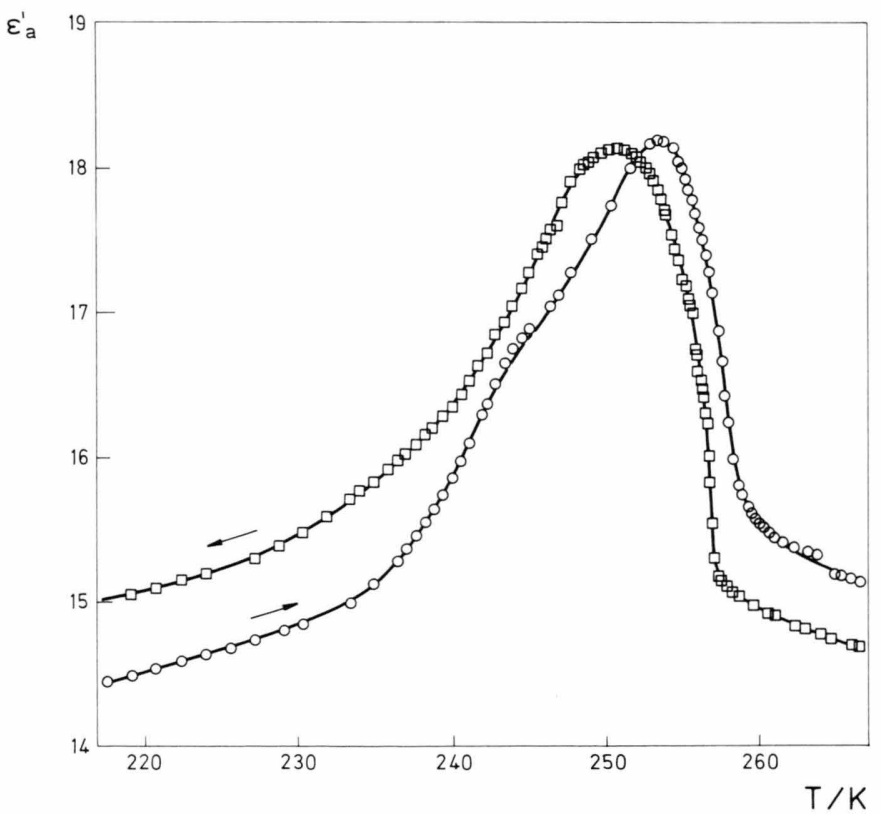

Fig. 2. Electric permittivity versus temperature for TEABA along $a$-axis.

The temperature dependencies of $\varepsilon^{\prime}(T)$ and $\tan \delta(T)$ for TEABB along the $a$-axis are presented in Figs. 3 and 4 , respectively. On cooling $\varepsilon_{a}^{\prime}$ stays constant from $290 \mathrm{~K}$ to 265.5 (Fig. 3) and around $T_{c 1}=265 \mathrm{~K}$ jumps up to $\varepsilon_{a(\max )}^{\prime}=18$ and then drops within $4 \mathrm{~K}$ to a min- 


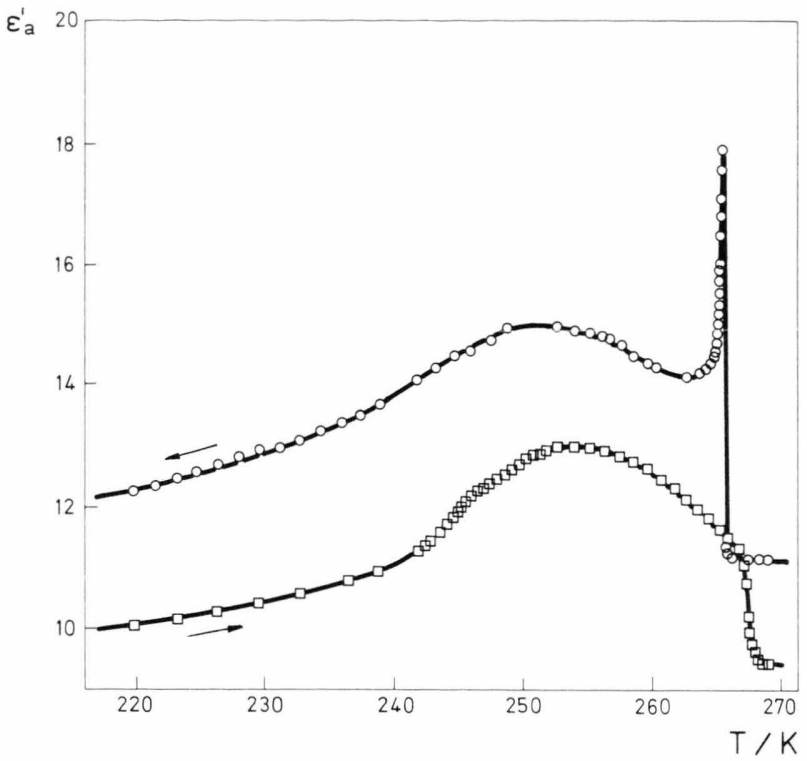

Fig. 3. Electric permittivity versus temperature for TEABB along $a$-axis.

imum $\left(\varepsilon_{a}^{\prime}=14.2\right)$. The anomaly of $\varepsilon_{a}^{\prime}(T)$ around $T_{c 1}$ on cooling is accompanied by a rather large anomaly of $\tan \delta_{a}$ (Fig. 4) in a very narrow temperature range of about $0.3 \mathrm{~K}$. It is worth noting that $\tan \delta_{a}$ reaches its maximum $\left(\tan \delta_{a(\max )}=1.2\right)$ at a slightly higher temperature $(0.1-0.15 \mathrm{~K})$ than $T_{c}\left(\varepsilon_{a(\max )}^{\prime}\right)$, and $\varepsilon_{a(\max )}^{\prime}$ corresponds to $\tan \delta_{a}=0.6$. On further cooling a broad maximum is observed around $T_{c 2}=248 \mathrm{~K}\left(\varepsilon_{a}^{\prime}=15\right)$ followed by a decrease of the permittivity down to $\varepsilon_{a}^{\prime}=12$ at $220 \mathrm{~K}$. No anomaly of $\tan \delta_{a}$ was observed around $T_{c 2}$. The temperature dependence of $\varepsilon_{a}^{\prime}$ is different on heating. The anomaly near $T_{c 2}$ is shifted by about $3 \mathrm{~K}$ to higher temperature and instead of the expected maximum a sharp drop of $\varepsilon_{a}^{\prime}(T)\left(\Delta \varepsilon_{a}^{\prime}=2\right)$ is observed near $T_{c 1}$. The anomaly of $\tan \delta_{a}$ was not observed.

The $\varepsilon_{c}^{\prime}(T)$ plot for TEABB in the vicinity of $T_{c 1}$ on cooling (Fig. 5) is similar to the $\varepsilon_{a}^{\prime}(T)$ plot but the maximum is much higher $\left(\varepsilon_{a(\max )}^{\prime}=30\right)$. Also in this case the anomaly of $\tan \delta_{c}$ near $T_{c 1}$ is large and $\varepsilon_{c(\max )}^{\prime}$ corresponds to $\tan \delta_{c}=0.8$. It should be noticed that no anomaly of $\varepsilon_{c}^{\prime}$ near $T_{\mathrm{c} 2}$ was found.

On heating (Fig. 6) near $245 \mathrm{~K}$ a faint anomaly is observed $\left(\Delta \varepsilon_{c}^{\prime}=1\right)$ which can be interpreted as the left wing of the broad peak at $T_{c 2}$, and on further heating a stronger one $\left(\Delta \varepsilon_{c}^{\prime}=2\right)$ near $268 \mathrm{~K}$ is visible. These changes of $\varepsilon_{c}^{\prime}(T)$ are accompanied by a small but distinct anomaly of $\tan \delta_{c}$ in the same temperature range.

The comparison of the temperature dependencies of $\varepsilon_{c}^{\prime}$ and $\varepsilon_{a}^{\prime}$ for TEABB suggests that also this compound

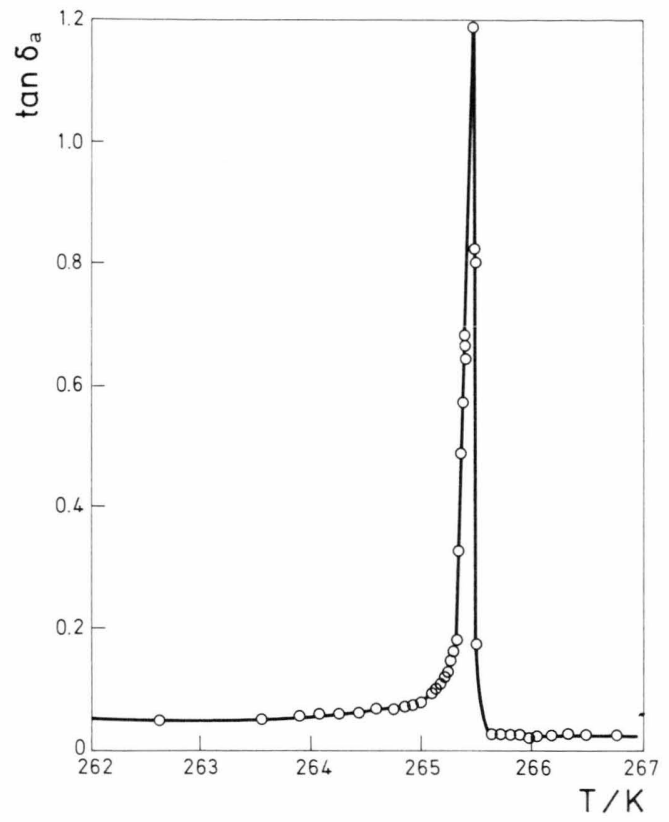

Fig. 4. $\tan \delta$ versus temperature for TEABB along $a$-axis in the vicinity of $T_{c 1}$ on cooling.

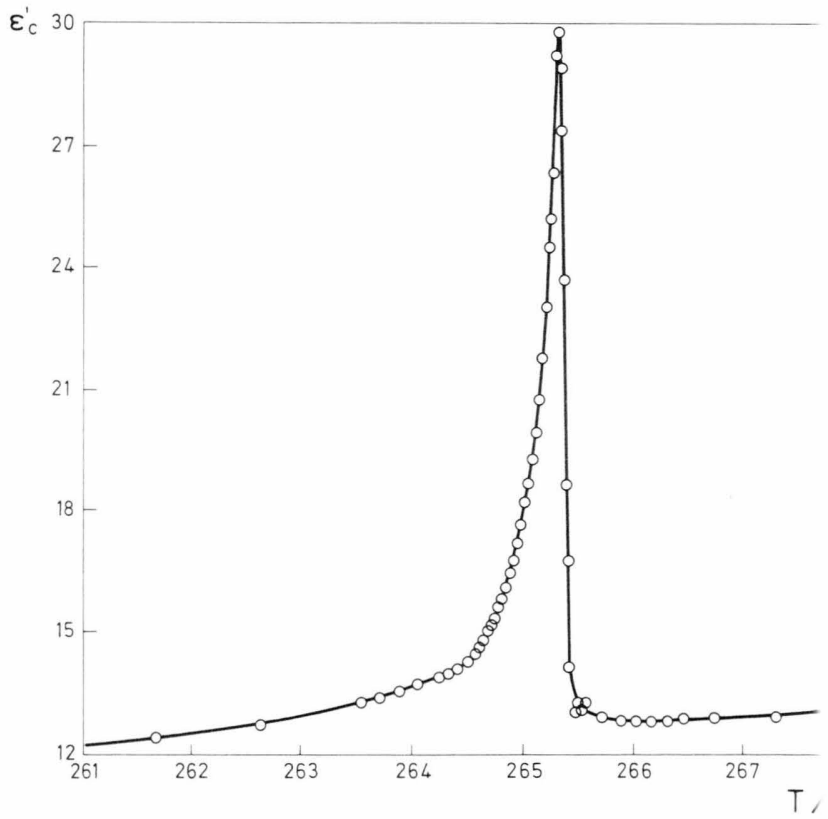

Fig. 5. Electric permittivity versus temperature for TEABB along $c$-axis in the vicinity of $T_{c 1}$ on cooling.

undergoes two structural phase transitions in the studied temperature range. These phase transitions are expressed by the temperature dependencies of the dielectric permittivity along the $c$ and $a$-axes, except of the anomaly round $T_{\mathrm{c} 2}$ which on cooling was not 


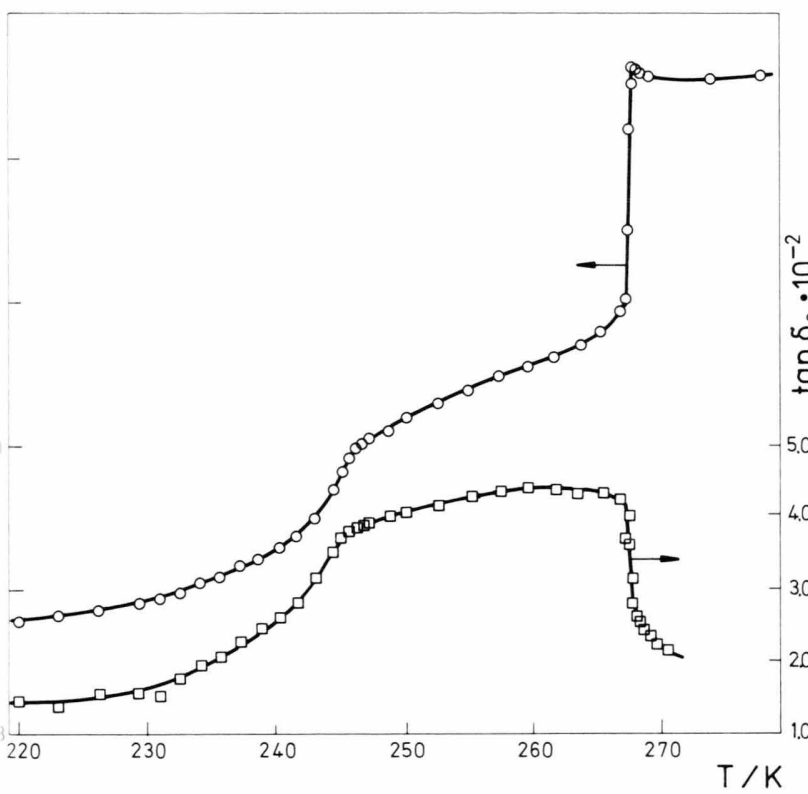

Fig. 6. Electric permittivity and $\tan \delta$ versus temperature for TEABB along $c$-axis on heating.

Table 1.

\begin{tabular}{llllll}
\hline Compound & $\begin{array}{l}T_{\mathrm{c}} \\
\mathrm{K}\end{array}$ & $\begin{array}{l}\Delta T_{\mathrm{c}} \\
\mathrm{K}\end{array}$ & $\begin{array}{l}\Delta H \\
\mathrm{~kJ} \mathrm{~mol}\end{array}$ & $\begin{array}{l}\Delta S \\
\mathrm{~J} \mathrm{~K}^{-1} \\
\cdot \mathrm{mol}^{-1}\end{array}$ & $\begin{array}{l}\Delta S / \text { cation } \\
\mathrm{J} \mathrm{Kol}^{-1}\end{array}$ \\
\hline (TEA) $\mathrm{mb}_{3} \mathrm{Sb}_{2} \mathrm{Br}_{9}$ & 258 & 0.5 & 3.1 & 12.2 & 4.1 \\
$(\mathrm{TEA})_{3} \mathrm{Bi}_{2} \mathrm{Br}_{9}$ & 265 & 2 & 4.4 & 16.7 & 5.6 \\
$(\mathrm{TEA})_{6} \mathrm{Bi}_{8} \mathrm{Cl}_{30}$ & 241 & 2 & 8.1 & 33.5 & 5.6 \\
\hline
\end{tabular}

observed in $\varepsilon_{c}^{\prime}(T)$. The observed hysteresis around $T_{c 1}$ $(2 \mathrm{~K})$ and the shape of the anomaly suggests first order character, while the order of the second transition, as in the case of the second anomaly for TEABA is not clear. The observed thermal hysteresis $(3 \mathrm{~K})$ suggest first order while the shape is typical for second order.

It should be added that the TEABB crystal breaks during the transition at $T_{c 1}$ on cooling, and the obtained values of $\varepsilon^{\prime}(T)$ at $T_{c 1}$ are not well reproducible.

The results of the calorimetric studies on TEABA and TEABB are presented in Table 1, where the results for a recently reported tetraethylammonium chlorobismuthate (TEA) ${ }_{6} \mathrm{Bi}_{8} \mathrm{Cl}_{30}$ [13] are included for comparison. In this crystal two closely lying $(0.5 \mathrm{~K})$ dielectric anomalies in a similar temperature range were observed. In contrast to the dielectric studies, the
DSC measurements show only one anomaly of first order for all studied compounds. It is interesting to note that the transition entropies per cation are comparable for all crystals under consideration (see Table 1).

The transition entropy is connected with the change in the number of states of disorder occupied statistically in the initial and final phases. In general the change of disorder of cations and anions contributes.

The structure of (TEA) ${ }_{6} \mathrm{Bi}_{8} \mathrm{Cl}_{30}$ crystal is known [13]. The crystal is composed of large $\mathrm{Bi}_{8} \mathrm{Cl}_{30}^{6-}$ anions and the TEA cations. The TEA cations were found to be disordered at room temperature. Taking into account the size of the anion it seems evident that the transition entropy for this crystal should be ascribed to the dynamics of the cations.

The transition entropies for all the TEA salts reported in Table 1 are comparable, which may suggest that the SPT at $T_{c 1}$ have the same origin. The transition entropies may testify to freezing of jumps of TEA cations between a limited number of potential energy minima.

In the crystal structures of the TEA salts, the TEA cations were found to be disordered at room temperature, e.g. $[2,3]$. In all cases the disorder is realized by the presence of two positions for all $C_{\alpha}$ carbon atoms. Such a disorder can be explained by assuming reorientational motion of the whole cation, flipping between two $\left(180^{\circ}\right.$ distant $)$ potential wells around a twofold axis (comprising the $\mathrm{N}$ atom and two opposite $C_{\beta}$ carbon atoms). The transition at $T_{c 1}$ would be thus connected with a freezing of such a hindered rotation. Let us mention that in this case $\Delta S=R \ln 2=5.76$ $\mathrm{J}$ mole ${ }^{-1} \mathrm{~K}^{-1}$ per cation.

The common feature of both crystals is the presence in a similar temperature range of two closely lying dielectric anomalies which are dielectrically active.

The high temperature anomaly at $T_{c 1}=265 \mathrm{~K}$ for TEABB appears as a peak observed along both directions, accompanied by a critical anomaly of $\tan \delta$. The magnitude of the observed effects and their temperature dependencies may be explained either by the presence of a low temperature polar phase or by a phenomenon connected with the breaking of the crystal at $T_{c 1}$. The latter explanation seems to be more probable. To inspect the presence of a low temperature ferroelectric phase, hysteresis loop studies (the field strength up to $4 \mathrm{kV} / \mathrm{cm}$ ) were undertaken. They gave a negative evidence, but if a ferroelectric phase below $T_{c 1}$ occurred it would be difficult to confirm it by this method be- 
cause the crystal breaks at $T_{c 1}$ which makes it impossible to use electric fields of higher strength.

The low temperature anomaly at $T_{c 2}=248 \mathrm{~K}$ for TEABB is similar to that at $T_{c 2}$ for TEABA with respect to the shape, though the maximum, is somewhat more diffused. Surprisingly this anomaly was not observed in $\varepsilon_{c}^{\prime}(T)$ on cooling.

The present DSC measurements, the X-ray diffraction study for (TEA) ${ }_{6} \mathrm{Bi}_{8} \mathrm{Cl}_{30}$ and the literature calorimetric data of similar crystals, e.g. from the (TEA) ${ }_{2} \mathrm{MX}_{4}$ family [4], indicate unambiguously the order-disorder mechanisms of a first order SPT.

Salts containing TEA cations were only two times studied by dielectric methods [9, 10]. Paper [9] relates to the $(\mathrm{TEA})_{2} \mathrm{MX}_{4}(\mathrm{M}=\mathrm{Co}, \mathrm{Zn}, \mathrm{Mn} ; \mathrm{X}=\mathrm{Cl}, \mathrm{Br})$ group. This study was carried out on polycrystalline samples, which precludes any quantitative comparison.

Paper [10] is devoted to (TEA) $\mathrm{FeCl}_{4}$ crystal. In this crystal two small dielectric anomalies at $T_{c 1}=236 \mathrm{~K}$ and $T_{c 2}=230 \mathrm{~K}$ were found. The common feature of (TEA) $\mathrm{FeCl}_{4}$ and our crystals is the presence of two dielectric anomalies in a similar temperature range (close to the transition temperatures of chlorobismuthate). The dielectric anomalies found in (TEA) $\mathrm{FeCl}_{4}$ crystal show, however, a different shape and are much smaller $\left(\Delta \varepsilon^{\prime}=0.1-0.4\right)$.

The dielectric properties of crystals from the family of halogenoantimonates and bismuthates of the general formula $\left[\left(\mathrm{CH}_{3}\right)_{n} \mathrm{NH}_{4-n}\right]_{3} \mathrm{M}_{2} \mathrm{X}_{9}$ (where $n=1,2,3,4$; $\mathrm{M}=\mathrm{Sb}, \mathrm{Bi} ; \mathrm{X}=\mathrm{Cl}, \mathrm{Br}$ ) seem, to be closely related to their crystal structure. The anionic sublattice of mono-, di- and trimethylammonium crystals is composed either of one dimensional stacks [14] or two dimensional layers [15] formed by $\mathrm{MX}_{6}$ octahedra, while in the tetramethylammonium salts the anions are composed of two face sharing $\mathrm{M}_{2} \mathrm{X}_{9}^{3-}$ octahedra [16]. It seems that the $\mathrm{N}^{+}-\mathrm{H} \cdots \mathrm{X}$ hydrogen bonds formed in mono-, di- and trimethylammonium salts

[1] R. Jakubas and L. Sobczyk, Ferroelectrics 78, 69 (1988).

[2] G. de Ashutosh and N. R. Chaudhuri, Thermochim. Acta 108, 97 (1986).

[3] J. H. Loehlin and A. Kvick, Acta Cryst. B 34, 3488 (1978).

[4] D. S. Brown, F. W. B. Einstein, and D. G. Tuck, Inorg. Chem. 8(1), 14 (1969).

[5] H. Ketata, M. H. Ben Ghozlen, A. Daoud, and I. Pabst, phys. stat. sol. (a) 103, 73 (1987).

[6] R. Navarro, J. A. Puertolas, E. Palaccio, and D. Gonzales, J. Chem. Therm. 20, 373 (1988).

[7] E. C. Reynhard and J. P. S. Rash, J. Mag. Resonance 42, $88(1981)$

[8] W. H. J. de Beer and A. M. Heyns, Spectr. Acta 37 A, 12 , 1099 (1981). play some role in the stabilizing of the polyanionic structures. Such hydrogen bonds are absent in the case of tetraalkylammonium salts. On the other hand the anionic sublattice does not change on going from the tetramethyl to the tetraethylammonium salts. The dielectric properties of these crystals are similar (small dielectric anomalies below room temperature).

\section{Conclusions}

1. Two dielectric anomalies were found for all the studied crystals in a similar temperature range.

2. The high temperature anomalies were confirmed by a DSC method. The transition entropies per cation are comparable for all the crystals suggesting a simple order-disorder mechanism of the transitions. It may relate to a freezing of $180^{\circ}$ jumps of the TEA cations between two potential energy minima around the twofold axis.

3. The high dielectric anomaly which accompanies the transition at $T_{c 1}$ in TEABB and its character of temperature dependence suggests either the presence of a polar low temperature phase or may be due to the hoping of the uncompensated charge related to the breaking of the crystal at $T_{c 1}$. The latter explanation seems to be more probable.

4. The low temperature anomalies for both crystials are to a large extent similar. The shape of the anomalies suggests second order phase transitions, but the DSC studies did not confirm these transitions.

5. The substitution of the tetramethylammonium cations by larger tetraethylammonium ones does not influence substantially both the structure and the dielectric properties of halogenoantimonates and bismuthates. Some differences between the TEA and TMA salts most probably can be ascribed to the additional dynamics of the TEA cations related to the ethyl groups.

[9] A. Wolthuis, W. J. Huiskamp, L. J. de Jongh, and R. L. Carlin, Physica 142 B, 301 (1986).

[10] J. Mróz and Z. Czapla, Ferroelectrics 81, 1009 (1988).

[11] G. C. Allen and R. F. McMeeking, Inorg. Chimica Acta 23, 185 (1977).

[12] M. J. F. Leroy and G. J. Goetz, Bull. Soc. Chim. Fr. 3-4, I 120 (1979).

[13] J. Zaleski, T. Głowiak, R. Jakubas, and L. Sobczyk. J. Phys. Chem. Solids, accepted for publication.

[14] R. Jakubas, Z. Czapla, Z. Galewski, L. Sobczyk,. O. J. Żogał and T. Lis, phys. stat. sol. (a) 93, 449 (1986).

[15] M. Gdaniec, Z. Kosturkiewicz, R. Jakubas, and L. Sobczyk, Ferroelectrics 77, 31 (1988).

[16] R. Jakubas, Z. Galewski, L. Sobczyk, and J. Matuszewski, Ferroelectrics 88, 83 (1988). 